\title{
Software design and realization of the bidirectional communication system in the smart grid
}

\author{
Qi Xue ${ }^{1, a}$, Zhirong Liang ${ }^{1, b}$ and Ying $\mathrm{Du}^{1, \mathrm{c}}$ \\ ${ }^{1}$ Chinese people's Liberation Army Armored Forces Academy, Anhui, 233000, China \\ axueqihero@163.com, b476772954@qq.com, 693221379@qq.com
}

Keywords: smart grid; bidirectional communication system; software design.

\begin{abstract}
With the soaring development of economy, people's living standards have been gradually improved, fueling increasing demands for power resources. In response to the trend, the traditional grid is evolved into the smart grid. In the smart grid, the communication system adopts the bidirectional technique, which increases the capacity and speed of communication. Based on the bidirectional communication system of the smart grid, this paper employs the MVC design pattern to design the WEB service software to achieve interaction between users and electric power resources and information.
\end{abstract}

\section{Introduction}

There are many sensors in the smart grid. The function of sensors is to monitor users' electricity demands, and report the information to the grid system so as to efficiently guarantee the reliable operation of the smart grid. During the process, communication accuracy and timeliness between sensors and the grid is of vital importance. Many current communication techniques have prominent advantages and disadvantages. Compared with the traditional single-directional communication technique, the bidirectional communication technique boasts not only a huge capacity and a small time delay, but also high accuracy and timeliness, which can facilitate networking and maintenance.

\section{Introduction of the MVC design pattern}

MVC design pattern is a typical WEB design pattern, which compulsorily separates input, output and treatment modules of applications. During the compilation of software codes, codes of various modules are realized separately [1]. It is made up of three parts. First, view. Its function is to display treatment results of the application system to users. Besides, users' input requests are also received by this part. After treatment, results will be demonstrated to users. Second, model. Its function is to treat users' requests and return the treatment results. This part is a core link of the treatment service process. Third, controller. Its function is to connect the view part and the model part together. After users input demands into the view, the controller will analyze users' demands, and choose the proper model based on result analysis to treat users' demands. In terms of functions and structure, the three parts of the MVC design pattern are independent from each other to realize each other's functions. When the MVC design pattern is employed for any design task, a model is corresponding to a view or multiple views. Thanks to the controller, flexibility of code compilation is improved.

\section{Bi-directional communication system software design based on the MVC design pattern}

\section{System model.}

The bi-directional communication system model of the smart grid features a three-tier framework, namely the perception layer, the network layer and the application layer. Every layer has different functions. The realization of functions of various layers and the information transmission between adjacent layers ensure system's stable operation. In the perception layer, there are multiple wireless sensor nodes, which mainly function to collect and transmit the perceptual information of electric power resources. Wireless sensor nodes are made up of four modules. First is the perception module, 
which can further be divided into sensor' s sub-module and AD/DC sub-module. Second is the treatment module, which can further be divided into the sub-module of the processor and the memory. Third is the wireless module, which can further be divided into Internet and other devices, whose functions mainly include receiving and sending information of electric power resources. The application layer consists of two parts, namely the WEB server and the database server, whose major functions include storing and displaying information of electric power resources.

\section{Overall design.}

First, overall framework design. In the current application development, J2EE technique is widely used. However, the technique features a huge coding quantity. While controlling different system layers, it is relatively complex. In this paper, the MVC design pattern is employed to solve problems facing J2EE technique. To the end, B/S framework is adopted during the design of WEB service software so as to combine J2EE and the MVC design model. The overall framework of the WEB service software includes the browser, the WEB server, the application server and the database, of which the WEB server is the design focus. During the design of the view part, JSP technique and Java Bean technique are employed; during the design of the model part, JavaBean technique and EJB modules are employed; during the design of the controller part, Servlet is employed [2].

Second, design of functional modules. The WEB server mainly serves relevant personnel in grid enterprises. Therefore, there are four functional modules, including the logging module, the registration module, the data checking module and the function display model.

\section{Module design and realization.}

First, design and realization of the data checking module. Personnel can check information of corresponding electric power resources obtained by certain sensor of certain region at certain moment through the model. At the same time, the information can be displayed through the curve chart on the WEB page, so personnel can accurately and quickly get the demand information directly [3]. Users can input corresponding requests through the browser. Besides, time, place and sensor nodes can be input. The data checking module can package the information and conduct corresponding checking in the database. If the corresponding data can be directed check, the curve chart will display it on the WEB page. If corresponding data cannot be checked, the system will give the prompt that there is no required data information in the form of the prompt dialog box.

Second, design and realization of the functional demonstration module. The module mainly functions to achieve real-time collection of data and network coding. Information collection of sensor nodes features real-time dynamic collection. According to the collected information, the function demonstration page will conduct a real-time update of tables and curves. The interval for the module to invoke the latest information from the data is $10 \mathrm{~s}$. At the same time, the data information is converted into the binary data, which are displayed in the table on the module page. Personnel can observe the information in the table, analyze the consistency between the node data and the broadcasting data of the relay node. If the two are consistent with each other, it suggests that there is network coding.

\section{Database design and realization.}

Database is an ordinary approach for data storage. The major reason is that databases boasts a good expandability, a low redundancy and structured display and independent existence of data. Besides, databases can well meet users' demands of data storage and treatment. The current database management systems can be divided into many categories. During software design, this paper adopts the SQL Server database. The functions of the database are relatively complete. During data operation, it can provide many system categories, and during the application development process, some common demands can be efficiently met. At present, it has been widely applied to the application development and the data storage. The database of the WEB service software contains five tables. First is Users' Information Table, which is used to store users' information. Second is Node 1 Table, which is used to store the electric power information of Node 1 . Third is Node 2 Table, which is used to store the electric power information of Node 2. Fourth is Node 3 Table, which is used to save the electric power information of Node 3. Fifth is the Node Xor Table, which is used to store 
the electric power information of source nodes and relay nodes during the demonstration of network's coding functions [4]. Among them, the structure of Node 1, Node 2 and Node 3 Table contains three parts with ID standing for the node serial number; receive-time for the time taken for the data to be stored in the database; temperature for the binary temperature information results.

\section{Conclusions}

Through the design and realization of the WEB service software, functions including SOCKET communication, data communication and graph visualization can be efficiently realized. In this way, users can check their electric power demand information by logging on the system through the computer. Thus, interaction between users and electric power information is materialized.

\section{References}

[1] Cao J W, Wan Y X, Tu G L, et al. Information system architecture for smart grids[J]. Chinese Journal of Computers, 2013, (01): 143-167.

[2] Xu X, Zhao X Y, Xiang M, et al. Design of heterogeneous network communication adapter used for smart grid[J]. Automation \& Instrumentation, 2013, (07): 31-34.

[3] Chen L, Lv F C, Xie Q, et al. Research of real-time energy measurement and bidirectional communication smart meter[J]. Proceedings of the CSEE, 2011, (S1): 94-99.

[4] Zhang Q, Sun Y G, Yang T, et al. Applications of wireless sensor networks in smart grid[J]. Electric Power, 2010, (06): 31-36. 